\title{
Secreted Peptide PIP1 Induces Stomatal Closure by Activation of Guard Cell Anion Channels in Arabidopsis
}

\section{OPEN ACCESS}

Edited by:

Shanjin Huang,

Tsinghua University, China

Reviewed by:

Yun Xiang,

Lanzhou University, China

Juan Xu,

Zhejiang University, China

${ }^{*}$ Correspondence:

Donghua Chen

donghua7103@sdu.edu.cn

Wei Zhang

weizhang@sdu.edu.cn

Specialty section:

This article was submitted to

Plant Cell Biology,

a section of the journa

Frontiers in Plant Science

Received: 12 March 2020 Accepted: 23 June 2020

Published: 08 July 2020

Citation:

Shen J, Diao W, Zhang L, Acharya BR, Wang $M$, Zhao $X$, Chen $D$ and Zhang W (2020) Secreted Peptide PIP1 Induces Stomatal Closure by Activation of Guard Cell Anion Channels in Arabidopsis.

Front. Plant Sci. 11:1029. doi: 10.3389/fp/s.2020.01029

\author{
Jianlin Shen ${ }^{1}$, Wenzhu Diao ${ }^{1}$, Linfang Zhang ${ }^{1}$, Biswa R. Acharya ${ }^{2}$, Mei Wang ${ }^{1}$, \\ Xiangyu Zhao ${ }^{3}$, Donghua Chen ${ }^{1 *}$ and Wei Zhang ${ }^{1 *}$ \\ ${ }^{1}$ Key Laboratory of Plant Development and Environmental Adaption Biology, Ministry of Education, School of Life Science, \\ Shandong University, Qingdao, China, ${ }^{2}$ College of Natural and Agricultural Sciences, University of California Riverside, \\ Riverside, CA, United States, ${ }^{3}$ State Key Laboratory of Crop Biology, College of Life Sciences, Shandong Agricultural \\ University, Tai'an, China
}

Plant stomata which consist of a pair of guard cells, are not only finely controlled to balance water loss as transpiration and $\mathrm{CO}_{2}$ absorption for photosynthesis, but also serve as the major sites to defend against pathogen attack, thus allowing plants to respond appropriately to abiotic and biotic stress conditions. The regulatory signaling network for stomatal movement is complex in nature, and plant peptides have been shown to be involved in signaling processes. Arabidopsis secreted peptide PIP1 was previously identified as an endogenous elicitor, which induced immune response through its receptor, $R L K 7$. PIP1-RLK7 can activate stomatal immunity against the bacterial strain Pst DC3118. However, the molecular mechanism of PIP1 in stomatal regulation is still unclear and additional new factors need to be discovered. In this study, we further clarified that PIP1 could function as an important regulator in the induction of stomatal closure. The results showed that PIP1 could promote stomata to close in a certain range of concentrations and response time. In addition, we uncovered that PIP1-RLK7 signaling regulated stomatal response by activating S-type anion channel SLAC1. PIP1-induced stomatal closure was impaired in bak1, mpk3, and mpk6 mutants, indicating that BAK1 and MPK3/MPK6 were required for PIP1-regulated stomatal movement. Our research further deciphered that OST1 which acts as an essential ABA-signaling component, also played a role in PIP1-induced stomatal closure. In addition, ROS participated in PIP1induced stomatal closure and PIP1 could activate $\mathrm{Ca}^{2+}$ permeable channels. In conclusion, we reveal the role of peptide PIP1 in triggering stomatal closure and the possible mechanism of PIP1 in the regulation of stomatal apertures. Our findings improve the understanding of the role of PIP1 in stomatal regulation and immune response.

Keywords: guard cells, secreted peptide, PIP1, stomatal closure, anion channel 


\section{INTRODUCTION}

Plant stomatal pores are formed by pairs of specialized epidermal guard cells and serve as major gateways to modulate gas exchange for photosynthesis and transpirational water loss (Bergmann and Sack, 2007). Stomata are also the major entry sites for different kinds of pathogens. Plants frequently suffer from various abiotic and biotic stresses during their life cycle, and stomata play an important role in allowing plants to respond appropriately to diverse environmental stimuli or defend against pathogen attack (Melotto et al., 2006; Kim et al., 2010; Blatt et al., 2017). The regulatory signaling pathway of stomatal movement is complex and a lot of elements can influence stomatal pore apertures, such as $\mathrm{CO}_{2}$, light, humidity, phytohormones, pathogens (Speth et al., 2009; Kollist et al., 2014; Murata et al., 2015). Among these factors, plant stress hormone abscisic acid (ABA) signaling has been studied extensively and shown to play a vital role in the regulation of stomatal movement (Wang et al., 2001; Raghavendra et al., 2010; Lee et al., 2013). ABA can trigger the activation of guard cell anion channels (e.g. SLAC1) and can result in the efflux of anions, which in turn reduces the turgor of guard cells to promote stomatal closure (Li et al., 2000; Cutler et al., 2010; Okamoto et al., 2013; Merilo et al., 2015). In addition, the cytosol reactive oxygen species (ROS) and $\mathrm{Ca}^{2+}$ are important second messengers which are elevated in response to ABA that in turn function as positive regulators in ABA-mediated stomatal closure (Pei et al., 2000; Kim et al., 2010; Suzuki et al., 2011; Singh et al., 2017).

There are numerous small signaling peptides have been discovered in plants and most of them do not have known function. Primarily, small signaling peptides have been found to play roles in plant growth and development (Wang and Fiers, 2010; Murphy et al., 2012; Czyzewicz et al., 2013; Dong et al., 2019). Additional findings also implicate that small signaling peptides can play roles in regulating stomatal movement and can response to abiotic or biotic stress (Wang et al., 2016; Takahashi et al., 2018; Yu et al., 2018; Zheng et al., 2018; Qu et al., 2019). flg22 is a 22-amino acid peptide, which is derived from the bacterial flagellin protein (Monaghan and Zipfel, 2012). In Arabidopsis, flg22 can be recognized by the receptor kinase FLS2 in plasma membrane and then FLS2 interacts with BRI1-associated kinase 1 (BAK1) to form an active complex by phosphorylation (Chinchilla et al., 2007; Sun et al., 2013). Besides, another receptor-like kinase, BOTRYTISINDUCED KINASE 1 (BIK1), is a direct substrate of the FLS2BAK1 complex which phosphorylates the NADPH oxidase that in turn induces the production of ROS (Li et al., 2014). flg22-FLS2 pathway can induce a transient elevation of cytosolic $\mathrm{Ca}^{2+}$ and the production of ROS, which ultimately induces stomatal closure to prevent bacterial invasion (Melotto et al., 2006). It is noteworthy that OPEN STOMATA 1 (OST1) and SLAC1 are essential factors for ABA-induced stomatal closure which have been also shown to mediate the flg22-regulation of stomatal closure. In fact, OST1 or second messengers (e.g. ROS or $\mathrm{Ca}^{2+}$ ) can activate SLAC1 to promote stomatal closure (Geiger et al., 2009; Vahisalu et al., 2010; Joshi-Saha et al., 2011). These findings suggest that there is crosstalk between ABA pathway and small peptide-mediated regulation of stomatal movement. In addition, MITOGENACTIVATED PROTEIN KINASE MPK3/MPK6 are known to play roles in plant immune response (Mao et al., 2011; Meng et al.,
2013; Su et al., 2017) and in small peptide-induced stomatal closure (Zhang et al., 2019).

Arabidopsis secreted peptide PIP1 can be recognized by RLK7, which is plasma membrane-localized LRR-RLK (Pitorre et al., 2010), and can amplify immunity response (Hou et al., 2014). A recent report indicates that PIP1 cooperates with salicylic acid to regulate stomatal immunity in Arabidopsis thaliana (Hou et al., 2019). In this study, we further confirmed that PIP1 could participate in the induction of stomatal closure. PIP1 could promote stomatal closure in a time- and dose-dependent manner. Moreover, anion channel SLAC1 played an important role in PIP1RLK7 signaling pathway. We further showed that $B A K 1, M P K 3 / 6$, and an important signaling element of the ABA-pathway OST1 were required for PIP1-induced stomatal closure. In addition, our research revealed that $\mathrm{ROS}$ and $\mathrm{Ca}^{2+}$ channels acted as the downstream components in PIP1-RLK7 signaling. Altogether, our study demonstrated the mechanism of PIP1-induced stomatal closure to some extent, and provided clues to delineate signal transduction pathways mediated by PIP1 in stomatal regulation and stress response.

\section{MATERIALS AND METHODS}

\section{Plant Materials and Growth Conditions}

We used two wild type ecotypes of $A$. thaliana in this study: Columbia-0 (Col-0) and Landsberg-0 (Ler-0). Col-0 was the background of PIP1 over-expression lines (PIP1-OE and PIP1OE2), and mutants $r l k 7$ (rlk7-2, SALK_083114) (Hou et al., 2019), bak1 (bak1-4, SALK_116202) (Hou et al., 2014), bik1 (Zhang et al., 2010; Hou et al., 2014), mpk3/mpk6 (SALK_151594/SALK_073907) (Xu et al., 2014; Zhang et al., 2019), slac1-1, slac1-3, and rbohD/F (CS9558) (Shen et al., 2017). But ost1-1 and ost1-2 were Ler-0 ecotype background. As for seedling growth, seeds were surface-sterilized with $75 \%$ ethanol for $3 \mathrm{~min}$, then $95 \%$ ethanol for $1 \mathrm{~min}$, and followed air-dried before use. The sterilized seeds were subsequently plated on half-strength Murashige and Skoog (1/2 MS) solidified medium (containing $1 / 2 \mathrm{MS}$ salts, $1 \% \mathrm{w} / \mathrm{v}$ sucrose, and $0.7 \% \mathrm{w} / \mathrm{v}$ agar, $\mathrm{pH}$ 5.7) and then vernalized for 3 days at $4^{\circ} \mathrm{C}$. After vernalization, seeds were transferred to a growth chamber (8-h light/16-h dark cycle, 100 $\mu \mathrm{mol} \mathrm{m} \mathrm{s}^{-2}$ light, $70 \%$ relative humidity, a temperature regime of $22^{\circ} \mathrm{C} \pm 2{ }^{\circ} \mathrm{C}$ day $/ 18^{\circ} \mathrm{C} \pm 2{ }^{\circ} \mathrm{C}$ night) for 1 week further growth. Then the seedlings were transplanted to pots containing soil mixture (vermiculite: rich soil, 1:2, v/v).

\section{Peptide Synthesis}

According to the report (Hou et al., 2014), the peptide PIP1 used in this research was synthesized by Sangon Biotech Company (Shanghai, China) and the purity level of PIP1 was $98 \%$. The sequence of peptide is shown from $\mathrm{N}$ terminus to $\mathrm{C}$ terminus as follows: RLASG-Hyp-SPRGPGH.

\section{Stomatal Closure Experiment}

Stomatal apertures were measured as described previously with slight modification (Li et al., 2016). Fully expanded rosette leaves from about 4-week-old plants of every genotype were harvested for stomatal closure assay. Detached leaves were incubated in 
closure buffer $\left(1 \mathrm{mM} \mathrm{CaCl}_{2}, 20 \mathrm{mM} \mathrm{KCl}, 5 \mathrm{mM}\right.$ MES-KOH, $\mathrm{pH}$ 6.15 ) for $2.5 \mathrm{~h}$ in light. Then leaves were treated with PIP1, $\mathrm{H}_{2} \mathrm{O}_{2}$ or Pst DC3118 bacterial suspension (the final concentration of $\left.10^{8} \mathrm{cfu} / \mathrm{ml}\right)$ for the indicated time, and non-bioactive PIP1 (hyperthermia inactivation) was the control of PIP1 treatment and water was used as the control of Pst DC3118 or $\mathrm{H}_{2} \mathrm{O}_{2}$. Subsequently, abaxial epidermal strips were peeled away by tweezers to make slides and placed on a light microscope (Olympus SZX16) to photograph randomly. The stomatal pore widths and lengths were measured using Image J (version: 1.37, https://imagej.nih.gov/ij/), and the stomatal aperture was calculated as the ratio of the inner pore width/pore length of each pair of stomata (Yang et al., 2017). All experiments were repeated for three independent biological replicates, and no less than 40 guard cells were measured for every sample. Statistical analyses were performed using a One-way ANOVA followed by the significant difference test.

\section{Quantitative Real-Time PCR}

The quantitative real-time PCR (qPCR) was used to analyze the transcript levels of PIP1 and SLAC1 in response to treatment. Briefly, $A$. thaliana 15 -day-old seedlings grown on $1 / 2 \mathrm{MS}$ solidified agar plates were transferred to $1 / 2 \mathrm{MS}$ liquid medium for $24 \mathrm{~h}$ of incubation. Then $10 \mu \mathrm{M}$ PIP1 or Pst DC3118 bacterial suspension (the final concentration of $10^{8} \mathrm{cfu} / \mathrm{ml}$ ) was added to the medium and incubated for another hour. After indicated treatment time, seedlings were harvested and frozen quickly in liquid nitrogen. The transcription of PIP1 was also identified by qPCR in the overexpression lines (PIP1-OE and PIP1-OE2) (Hou et al., 2014). Total RNA was isolated from seedlings using TRIzol reagent (Roche, Switzerland) and cDNA was synthesized using the Revert Aid First Strand cDNA Synthesis Kit (Thermo Fisher, USA). qPCR experiments were performed using the CFX96 Touch $^{\text {TM }}$ Real-Time PCR Detection system (Bio-Rad, Hercules, CA, USA) which based on SYBR Premix Ex Taq mix (Roche) with gene specific primers. The internal control was ACTIN2. All the quantitative analysis was repeated for three independent biological replicates. The primer sequences used are shown as follows: ACTIN2 forward primer, $5^{\prime}$ GGTAACATTGTGCTCAGTGGTGG-3', ACTIN2 reverse primer, 5'-AACGACCTTAATCTTCATGCTGC-3'. PIP1 forward primer, 5'-AATCGGGAGAATGGAAGTGC-3', PIP1 reverse primer, 5' GACGCCAAACGCTGAAAC-3'. SLAC1 forward primer, 5' CCGGGCTCTAGCACTCA-3', SLAC1 reverse primer, 5' TCAGTGATGCGACTCTT-3'.

\section{Guard Cell Isolation and Electrophysiology}

Arabidopsis guard cell protoplasts were isolated according to Zhang et al. (2008) with some modifications. Briefly, the Arabidopsis abaxial epidermis were peeled from 12 to 14 expanded young leaves of 4-week-old plants. Then, all collected epidermis were blended in about $500 \mathrm{ml}$ distilled water for $28 \mathrm{~s}$ and filtered through a 100- $\mu$ m nylon mesh. Subsequently, the peels were transferred into $2 \mathrm{ml}$ enzyme solution I ( $0.7 \%$ Cellulysin cellulase, $0.1 \%$ PVP-40, and $0.25 \%$ BSA in 55\% basic solution ( $5 \mathrm{mM} \mathrm{MES,} 0.5$ $\mathrm{mM} \mathrm{CaCl}_{2}, 0.5 \mathrm{mM} \mathrm{MgCl}_{2}, 0.5 \mathrm{mM}$ ascorbic acid, $10 \mu \mathrm{M} \mathrm{KH}_{2} \mathrm{PO}_{4}$, $0.55 \mathrm{M}$ sorbitol, $\mathrm{pH}$ 5.5). The peels were digested in a shaking water bath at $80 \mathrm{rpm}$ for $30 \mathrm{~min}$ at $25^{\circ} \mathrm{C}$. Another 2 -ml basic solution was added to enzyme solution $\mathrm{I}$, and shaking was continued for a further
$10 \mathrm{~min}$. After that, the partially digested peels were filtered through a $100-\mu \mathrm{m}$ nylon mesh and put into $2 \mathrm{ml}$ of enzyme solution II, which contained $1.5 \%$ Onozuka cellulase RS, $0.02 \%$ cellulase Y-23, and $0.25 \%$ BSA in $100 \%$ basic solution. Then digestion of the peels continued by shaking at $60 \mathrm{rpm}$ for at least $20 \mathrm{~min}$. After digestion, these peels were collected and filtered through $30-\mu \mathrm{m}$ nylon mesh. The guard cell protoplasts were obtained by centrifuging at $800 \mathrm{rpm}$ for $5 \mathrm{~min}$ and washed twice by basic solution.

The whole-cell mode patch clamp experiment was performed as described previously (Pei et al., 1997; Pei et al., 2000; Wang et al., 2001; Acharya et al., 2013). To record the S-type anion channel currents, the bath solution contained $30 \mathrm{mM} \mathrm{CsCl}, 2$ $\mathrm{mM} \mathrm{MgCl} 2,1 \mathrm{mM} \mathrm{CaCl}$, and $10 \mathrm{mM} \mathrm{MES} \mathrm{(pH} \mathrm{5.6)} \mathrm{and} \mathrm{the}$ pipette solution contained $150 \mathrm{mM} \mathrm{CsCl}, 2 \mathrm{mM} \mathrm{MgCl}$, $6.7 \mathrm{mM}$ EGTA, $3.35 \mathrm{mM} \mathrm{CaCl}_{2}$, and $10 \mathrm{mM}$ HEPES ( $\mathrm{pH}$ 7.5). The osmolarity of the solutions was adjusted respectively with sorbitol to 480 and $500 \mathrm{mOsm}$ for bath and pipette solutions. The ATP (MgATP, $10 \mu \mathrm{M})$ and GTP $(10 \mu \mathrm{M})$ were added to pipette solutions before use from stock solutions. To investigate PIP1 activation of $\mathrm{Ca}^{2+}$-permeable $\mathrm{I}_{\mathrm{Ca}}$ channels, the pipette solution contained $10 \mathrm{mM}$ $\mathrm{BaCl}_{2}, 4 \mathrm{mM}$ EGTA, $0.1 \mathrm{mM}$ DTT, and $10 \mathrm{mM}$ HEPES-Tris ( $\mathrm{pH}$ 7.1). $5 \mathrm{mM}$ NADPH was freshly added to the pipette solution before experiments. The bath solution contained $100 \mathrm{mM} \mathrm{BaCl}_{2}, 0.1 \mathrm{mM}$ DTT, and $10 \mathrm{mM}$ MES-Tris (pH 5.6). Osmolarity was adjusted to 500 and $485 \mathrm{mOsm}$ for the pipette solution and the bath solution respectively with $\mathrm{D}$-sorbitol.

The anion channel currents were recorded using the Axopath200B amplifier (Molecular Devices, Downingtown, PA, USA) after the whole-cell configuration was achieved. The holding potential was $+30 \mathrm{mV}$, and voltage steps were applied from -145 to $+35 \mathrm{mV}$ in $+30 \mathrm{mV}$ increments, with a duration of $60 \mathrm{~s}$ for every test voltage. For PIP1 treatment, guard cell protoplasts were exposed to $10 \mu \mathrm{M}$ PIP1 for $2 \mathrm{~h}$ before measurement and PIP1 was also added to both the bath and pipette solution. As to record $\mathrm{Ca}^{2+}$ channel currents, the whole-cell $\mathrm{Ca}^{2+}$ currents were recorded 5 min after achieving the whole-cell configuration. The holding potential was $-13 \mathrm{mV}$ and voltage ramps were from -200 to $+80 \mathrm{mV}$. PIP1 or $\mathrm{LaCl}_{3}$ was added to the bath solution. To acquire and analyze the anion or $\mathrm{Ca}^{2+}$ channel currents, pCLAMP software (version 10.2; Axon Instruments, Sunnyvale, CA, USA) was used, and SigmaPlot 12.0 (Systat Software, Richmond, CA, USA) was used to draw the current-voltage plots and for data analysis.

\section{RESULTS}

\section{PIP1 Peptide Can Promote Stomatal Closure in A. thaliana}

It is well known that stomata are the major entry sites for various pathogens, and PIP1 has been previously shown to function in plant immunity (Hou et al., 2014). Therefore, we found that exogenous treatment of Pst DC3118 bacteria, which is the COR deficient, could induce PIP1 expression (Figure 1). Besides, Pst DC3118 could close the stomata in wild type, and the stomatal apertures were much smaller in PIP1 over-expression lines than those of Col-0 (Figure 1 and Supplementary Figure S3). These results indicated that PIP1 could close stomata to restrict 

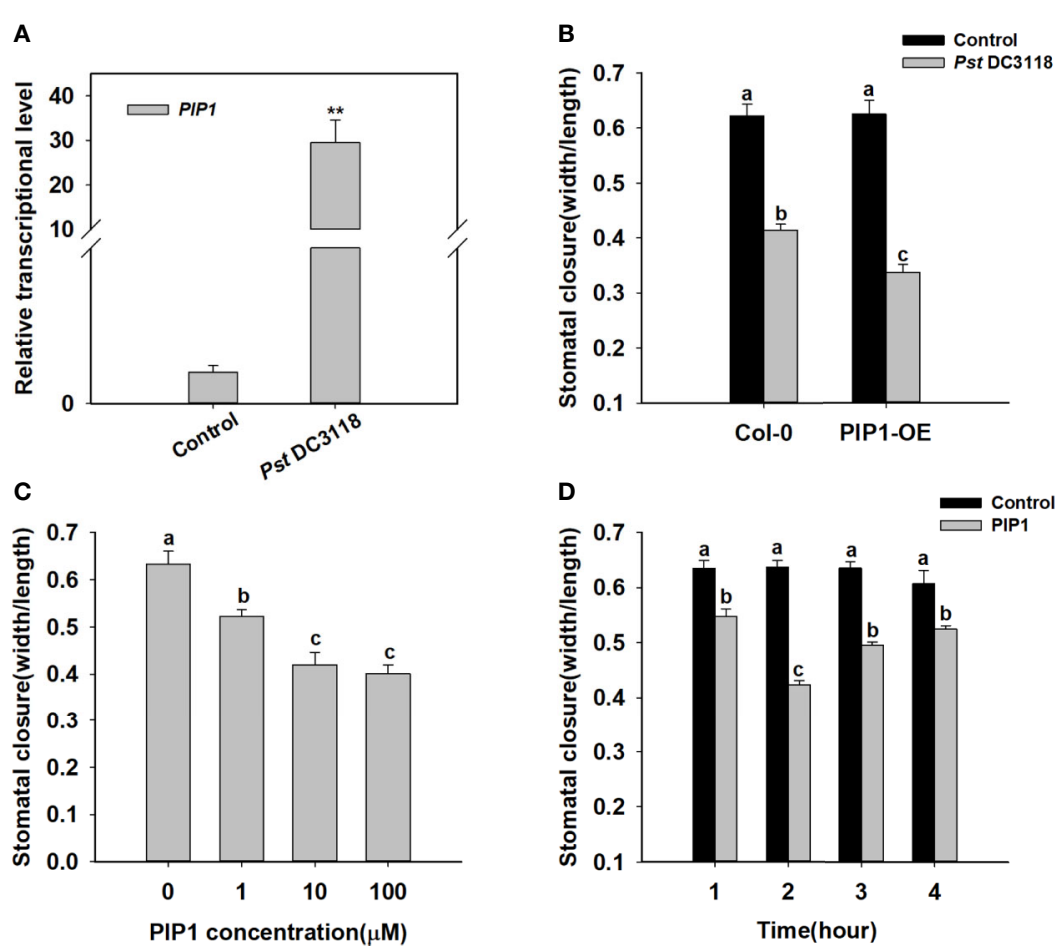

FIGURE 1 | PIP1 induces stomatal closure in Arabidopsis. (A) Induction of transcript level of PIP1 in response to Pst DC3118 treatment (the final concentration of $\left.10^{8} \mathrm{cfu} / \mathrm{ml}\right)$. Asterisks indicate significant differences between means $(\mathrm{P}<0.01)$. (B) Stomatal aperture in PIP1 overexpression plants by treating with $P$ st DC3118 (the final concentration of $\left.10^{8} \mathrm{cfu} / \mathrm{ml}\right)$. (C) Induction of stomatal closure by different concentrations of PIP1 peptides (0, 1 , 10, $\left.100 \mu \mathrm{M}\right)$. (D) Time course of stomatal closure in response to $10 \mu \mathrm{M}$ PIP1 treatment. In stomatal closure experiments, error bars indicate SE for three independent biological replicates. Different letters represent significant differences between groups using Holm-Sidak significant difference test after one-way ANOVA (P value $<0.05)$.

pathogen entry via a PIP1-dependent manner. To further confirm the function of PIP1 in stomatal movement, we synthesized PIP1 peptides as described by Hou et al. (2014) and conducted stomatal closure experiments. Firstly, we analyzed stomatal aperture by exogenous application of different concentrations $(0,1,10,100$ $\mu \mathrm{M})$ of PIP1 peptides. The result showed that the stomatal aperture decreased in size under higher concentrations of PIP1 until the 10 $\mu \mathrm{M}$ concentration (Figure 1). Additionally, we also found that PIP1 could induce stomatal closure in a time-dependent manner. As shown in Figure 1, the stomatal aperture did not change obviously without PIP1 exposure but decreased within 1 to $2 \mathrm{~h}$ upon $10 \mu \mathrm{M}$ PIP1 treatment, and the minimum aperture was observed at $2 \mathrm{~h}$ (Figure 1). Interestingly, the stomatal aperture began to recover at 3 $\mathrm{h}$ of PIP1 treatment. According to these results, we used $10 \mu \mathrm{M}$ PIP1 as the optimal concentration and $2 \mathrm{~h}$ as the optimal treatment time for our follow-up experiments. These results indicate that secreted peptide PIP1 of Arabidopsis can play a role in regulating stomatal closure as a signaling molecule and can function in dose- and timedependent manner.

\section{The Anion Channel SLAC1 Functions in PIP1-RLK7 Signaling}

Previous studies have shown that SLAC1 is the major component of S-type anion channels and plays an important role in ABA-, high $\mathrm{CO}_{2^{-}}$, flagellin-induced stomatal closing responses in guard cells (Vahisalu et al., 2008; Kim et al., 2010; Guzel Deger et al., 2015; Zhang et al., 2018). These facts prompt us to find out whether SLAC1 is also required for PIP1-induced stomatal closure. We found that exogenous PIP1 could improve the transcriptional level of SLAC1 (Figure 2). In the presence of exogenous PIP1, the stomatal aperture reduced obviously in Col-0, but did not in slac1 mutants: slac1-1 and slac1-3 (Figure 2). In addition, similar to PIP1, Pst DC3118 could induce the transcription of SLAC1 (Supplementary Figure S1). Moreover, the genetic result showed that the stomatal closure of slac1 mutants were hyposensitive to Pst DC3118 (Supplementary Figure S1). These findings suggest that SLAC1 may play an important role in PIP1-induced stomatal closure and the response to Pst DC3118.

The research has shown that PIP1-induced stomatal closure is RLK7 dependent (Hou et al., 2019). To explore the mechanism of PIP1-RLK7 pathway in the regulation of stomatal response, we also conducted patch clamp whole-cell recordings of S-type anion currents in Col-0 and $r l k 7$ guard cell protoplasts under control condition and treated with $10 \mu \mathrm{M}$ PIP1. The path clamp data showed that the size of anion currents of Col-0 guard cells increased when exposed to PIP1 (Figure 2C). However, the anion currents of guard cells from $r l k 7$ mutants failed to increase after addition of PIP1 (Figure 2C). In addition, SLAC1 transcripts did not change in rlk7 with PIP1 treatment (Supplementary Figure S2), which suggest RLK7 is essential 
A

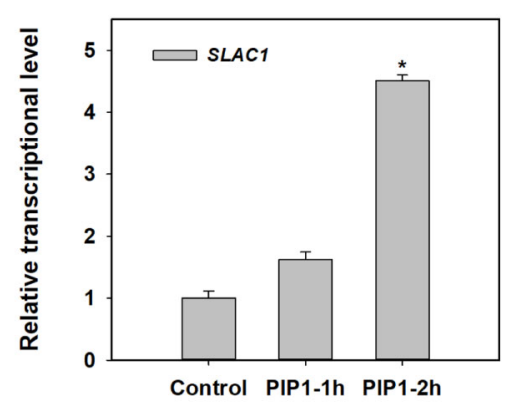

C

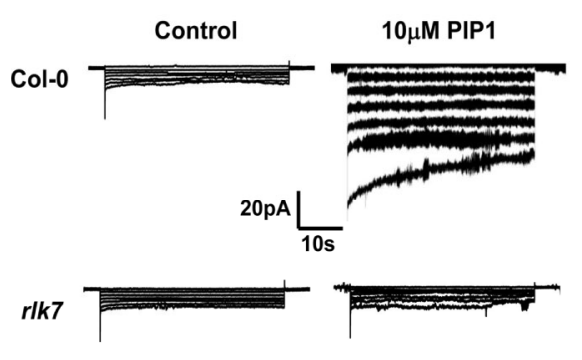

B

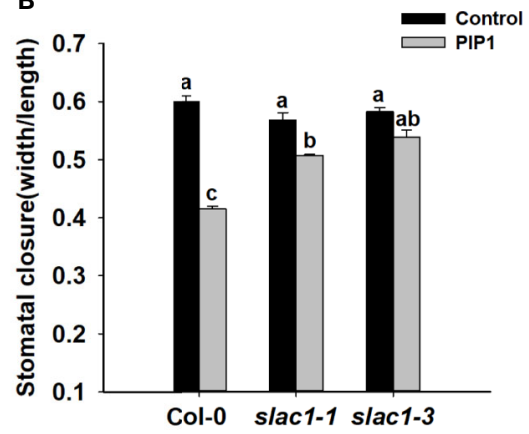

D

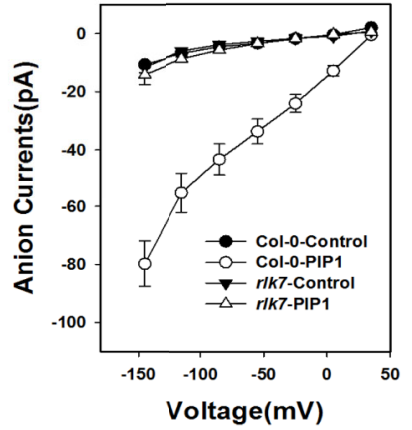

FIGURE 2 | SLAC1 plays an important role in PIP1-RLK7 signaling. (A) Evaluation of SLAC1 induction in response to $10 \mu M$ PIP1 by qPCR. Asterisks indicate significant differences between means ( $\left.{ }^{*}: \mathrm{P}<0.05\right)$. (B) Stomatal closure experiments in slac1 mutants (slac1-1 and slac1-3) in response to 10 $\mu$ M PIP1. Error bars indicate SE for three independent biological replicates. Different letters represent significant differences between groups after one-way ANOVA (P value $<0.05)$. (C) Patch clamp whole-cell recordings of the anion currents in Col-0 and rlk7 guard cell protoplasts with/without $10 \mu \mathrm{M}$ PIP1. (D) Current/voltage relationships of wholecell slow-type anion currents, as illustrated in (C). The numbers of guard cells measured were as follows: Col-0-Control, $n=11 ;$ Col-0-PIP1, $n=7 ;$ rlk7-Control, $n=$ 7; rlk7-PIP1, $\mathrm{n}=7$. Values are means $\pm \mathrm{SE}$.

for PIP1 induced SLAC1 transcription, and thus to facilitate the anion solutes efflux of guard cells to close stomata.

\section{BAK1, MPK3/6, and OST1 Take Part in PIP1-Induced Stomatal Closure}

The receptor kinase BAK1 and BIK1 often play an important role in peptide signaling or immune response by forming heteromeric co-receptor complexes with multiple LRR-RLK receptors (such as FLS2, PEPR1, and so on) (Chinchilla et al., 2007; Lu et al., 2010; Liu et al., 2013; Zheng et al., 2018). Additionally, BAK1 can regulate ABA-induced stomatal closure in guard cells (Shang et al., 2016). Previous report indicates that PIP1-RLK7 signaling is partially dependent on $B A K 1$, but independent of BIK1 (Hou et al., 2014). Therefore, we would like to test the possible functions of $B A K 1$ and $B I K 1$ in PIP1-induced stomatal closure. Firstly, we used genetic approach to further examine if $B A K 1$ and $B I K 1$ participated in PIP1-induced stomatal closure. The stomatal closure experiments of Col-0, bakl, and bik1 showed that the stomatal aperture of bakl mutants became insensitive to PIP1 when compared with Col-0 and $b i k 1$ (Figure 3). Moreover, consistent with the observing result in $r l k 7$, further data revealed that PIP1 could not activate SLAC1 transcription in mutant bak1 (Supplementary Figure S2), however, PIP1 could normally induce SLAC1 transcription in mutant bik1 (Supplementary
Figure S2). Our findings suggest that $B A K 1$ instead of $B I K 1$ may be required for PIP1-induced stomatal closure.

Published reports show that flg22-induced stomatal closure is compromised in $m p k 3$ and $m p k 6$ mutants (Montillet et al., 2013) and $M P K 3 / M P K 6$ mediate peptide-induced stomatal movement (Zhang et al., 2018). Since MPK3 and MPK6 have been shown to be activated upon PIP1 induction in Arabidopsis seedlings (Hou et al., 2014), thus we also tested the contributions of MPK3 and MPK6 to PIP1-induced stomatal closure. The result showed that PIP1-triggered stomatal closure was impaired in both $m p k 3$ and mpk6 mutants (Figure 3). Our finding indicate that MPK3 and MPK6 also serve as the downstream components in PIP1RLK7 pathway.

Stomatal movement is regulated by the phytohormone abscisic acid (ABA) (Lee and Luan, 2012). The ABA-triggered activation of guard cell anion channels results in the efflux of anions, which in turn promotes guard cells to close the stomata (Wang et al., 2013). In addition, we has established that SLAC1 plays a role in stomatal response in PIP1-RLK7 pathway. Therefore, we would like to explore the role of ABA signaling in PIP1-induced stomatal closure in guard cells. OST1, a SnRK2-type kinase, which has been shown to be critical signaling element for ABA-induced activation of S-type anion channels and ABA-induced stomatal closure (Joshi-Saha et al., 2011; Brandt et al., 2012). OST1 also 
A

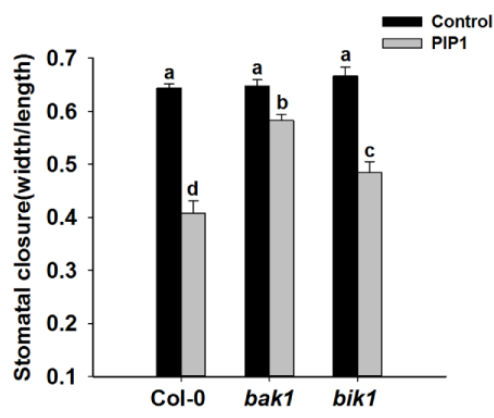

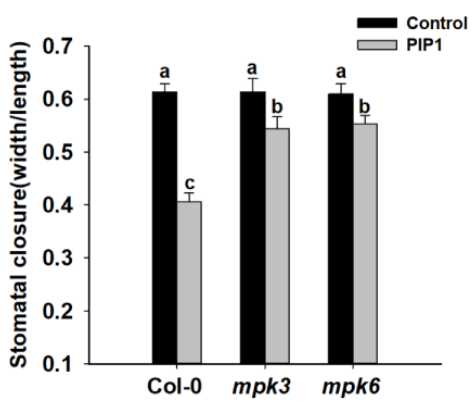

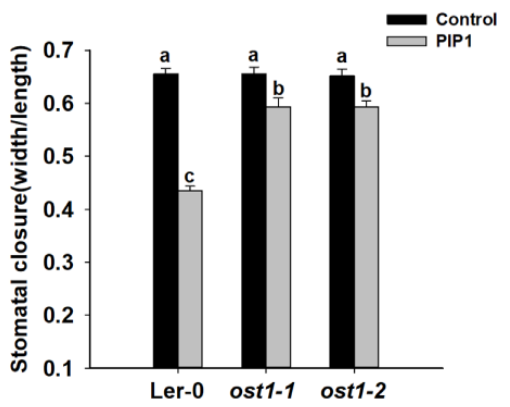

FIGURE 3 | BAK1, MPK3, MPK6, and OST1 are involved in PIP1-induced stomatal closure. (A-C) 10 MM PIP1-induced stomatal closure in bak1 and bik1 mutants (A), mpk3 and mpk6 mutants (B), ost1-1 and ost1-2 mutants (C). Values are means $\pm \mathrm{SE}(\mathrm{n}=3)$. All the experiments were performed in three independent biological replicates with similar results. Different letters indicate significant difference between groups after one-way ANOVA ( $P$ value $<0.05)$.

regulates flg22-induced stomatal closure and the activation of Stype anion channels (Guzel Deger et al., 2015). These facts prompted us to examine the possible function of OST1 in PIP1induced stomatal closure. The result showed that the stomatal apertures of OST1 mutants ost1-1 and ost1-2 were hyposensitive to PIP1 when compared with wild-type Ler-0 (Figure 3). Therefore, OST1 may also be required for PIP1-induced stomatal closure and OST1 may be a common node of PIP1 signaling and ABA signaling pathway in stomatal regulation.

\section{ROS Production and $\mathrm{Ca}^{2+}$ Signaling Are Required for PIP1-Regulated Stomatal Closure}

ROS, crucial signal molecules, participate in the regulation of stomatal movement (Purohit et al., 1994; Murata et al., 2015). PIP1 also has been reported to induce ROS production in adult leaves of Arabidopsis (Hou et al., 2014; Hou et al., 2019). AtRbohD and AtRbohF play essential roles in ROS production. Therefore, we genetically examined the stomatal response to PIP1 in rbohD/F double mutants, which fail to produce ROS. The result showed that $r b o h D / F$ double mutants were impaired in
PIP1-induced stomatal closure but stomatal aperture decreased again when treated with additional $\mathrm{H}_{2} \mathrm{O}_{2}$ (a form of ROS) (Figure 4). The result suggest that ROS is required for PIP1induced stomatal closure.

$\mathrm{Ca}^{2+}$ also functions as a key second messenger, which participates in the regulation of stomatal closure (Moscatiello et al., 2006) and Kwak et al. (2003) have reported that ROS can activate $\mathrm{Ca}^{2+}$-permeable channels and can produce concurrent cytosolic $\mathrm{Ca}^{2+}$ increase. In this study, we found that the ROS production participated in PIP1-induced stomatal closure, and it was needed to analyze whether $\mathrm{Ca}^{2+}$ also could be induced by PIP1. By using patch clamp, we detected a clear increase of $\mathrm{Ca}^{2+}$ currents through plasma membrane into cytoplasm after treatment with PIP1, but no obvious change of $\mathrm{Ca}^{2+}$ currents under control conditions was observed in guard cells (Figure 4B). The application of $\mathrm{LaCl}_{3}$, a calcium channel blocker, completely abolished the PIP1-induced $\mathrm{Ca}^{2+}$ influx (Figure 4B), indicating PIP1 can stimulate $\mathrm{Ca}^{2+}$ influx through activating the guard cell $\mathrm{Ca}^{2+}$ channels. In addition, our finding indicated that RLK7, receptor of PIP1, was required for the PIP1-activated $\mathrm{Ca}^{2+}$ currents (Figure 4C).
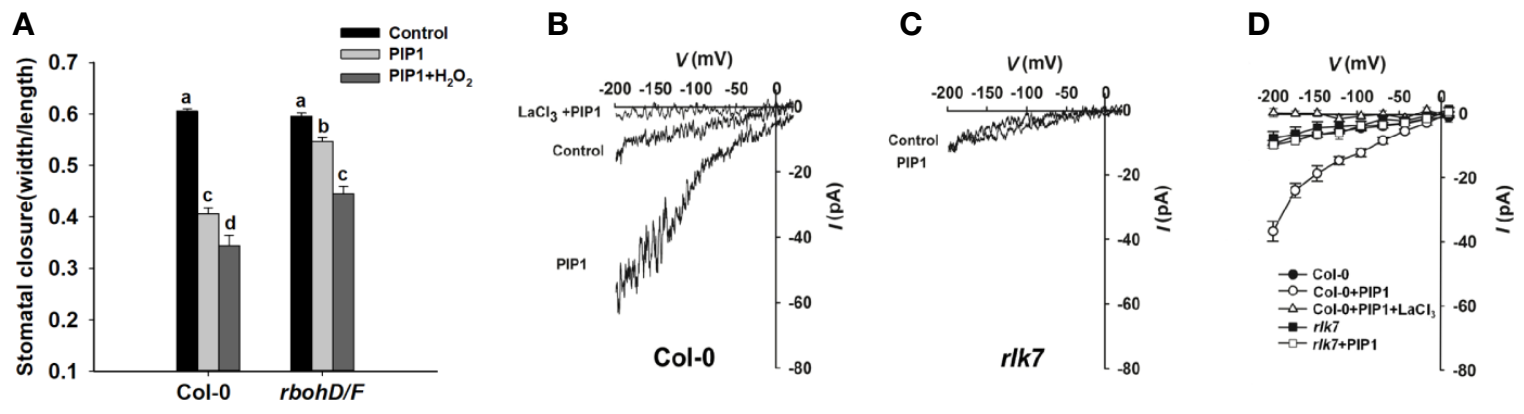

FIGURE 4 | ROS and $\mathrm{Ca}^{2+}$ signaling participate in PIP1-regulated stomatal closure. (A) The stomatal closure in Col-0 and double mutant rbohD/F exposed to 10 $\mu \mathrm{M}$ PIP1 or $10 \mu \mathrm{M}$ PIP1+100 $\mu \mathrm{M} \mathrm{H}_{2} \mathrm{O}_{2}$. Error bars indicate SE for three independent biological replicates. Different letters represent significant differences between groups after one-way ANOVA (P value < 0.05). (B, C) Typical whole cell recordings of $\mathrm{Ca}^{2+}$ currents in guard cell protoplasts isolated from Col-0 (B) and $r / k 7$ (C) with $10 \mu \mathrm{M}$ PIP1, $10 \mu \mathrm{M} \mathrm{PIP1+1} \mathrm{mM} \mathrm{LaCl}_{3}$. (D) Current/voltage curves of time-activated Ca ${ }^{2+}$ currents as indicated in (B, C). The number of guard cells measured as follows: Col-0 (control): 5; Col-0 (PIP1): 6; Col-0 (PIP1+LaCl $)$ : 6; rlk7 (control): 5; rlk7 (PIP1): 6. 


\section{DISCUSSION}

Plant stomata, consisting of a pair of guard cells, are dynamic structures that open or close to modulate gas exchange and water loss, and also allow plants to respond appropriately to diverse pathogens invasion. Therefore, stomata play essential roles in abiotic and biotic stress responses (Blatt et al., 2017). The regulation of stomatal movement is complex, and as time progresses, researchers discover new signaling elements which make the signaling networks of stomatal movement more complex (Chen et al., 2019; Sun et al., 2019). Recent years, in addition to plant growth and development, plant small signaling peptides have been implicated in stomatal aperture regulation (Li et al., 2014; Zheng et al., 2018; Qu et al., 2019). The Arabidopsis secreted peptide PIP1 plays a role in plant immune response, and the function of PIP1 is receptor RLK7-dependent (Hou et al., 2014). The PIP1-RLK7 pathway also functions with salicylic acid to regulate stomatal immunity (Hou et al., 2019). However, it is still unclear that the specific mechanism of PIP1-RLK7 signaling in stomatal regulation. In this study, we further identify new factors which play critical roles in PIP1-induced stomatal closure and the possible mechanism by which PIP1 regulates stomatal movement. This study also contributes to combine peptide-signaling with stomatal signal transduction pathway in guard cells.

We firstly further explored the impact of PIP1 on stomatal closure. Our findings showed that PIP1 could induce stomatal closure in a dose-dependent and a time-dependent manner (Figure 1C). The elevated transcription of PIP1 induced by Pst DC3118 (Figure 1) and the much smaller stomatal aperture of PIP1-OE lines exposed to Pst DC3118 (Figure 1 and Supplementary Figure S3), suggest that PIP1 can mimic the function of Pst DC3118 and can resist the pathogenic aggression through promoting stomatal closure. In addition, RLK7 acts as a PIP1 receptor in guard cells that activates stomatal immunity response upon PIP1 detection (Hou et al., 2019). Therefore, we further explored the mechanism of PIP1-RLK7 pathway induced stomatal closure. It is well known that the activation of S-type anion channels plays an important role in decreasing guard cell turgor and then leading to stomatal closure (Kim et al., 2010; Zhang et al., 2018; Munemasa et al., 2019). We found that SLAC1, an important S-type anion channel, mediated the PIP1-RLK7 signaling in regulating stomatal closure. The transcriptional level of SLAC1 was enhanced by treating with PIP1 (Figure 2) and the stomatal apertures of slac1-1 and slac1-3 mutants failed to respond to PIP1 (Figure 2). To obtain more concrete evidences for the involvement of SLAC1 in PIP1induced stomatal closure, we found that SLAC1 transcripts did not change in mutant $r l k 7$ with PIP1 treatment (Supplementary Figure S2). Additionally, we also tested the S-type anion currents in Col-0 guard cell protoplasts and found that PIP1 increased the size of anion currents (Figure 2C). However, PIP1 could not activate the S-type anion channels in rlk7 mutants (Figure 2C). The results confirm that PIP1-RLK7 signaling induce stomatal closure through the activation of S-type anion channel SLAC1.

In this study we has established that SLAC1 plays an important role in stomatal response to PIP1-RLK7 pathway, then we further explored the signaling mechanism for the activation of SLAC1 and sought key factors which transmitted the signal from PIP1-RLK7 to SLAC1. BAK1 belongs to the SERK family and acts as forming ligand-induced heteromers with multiple LRR-RLKs, including X subfamily of LRR-RLK BRI1 and XII subfamily of LRR-RLK FLS2 (Chinchilla et al., 2007; Wang et al., 2008). Previous study has found that PIP1-induced root inhibition is less predominant in bak1 mutants (Hou et al., 2014). Here, we also found that bakl was less sensitive to PIP1 for the induction of stomatal closure (Figure 3) and PIP1 could not induce the expression of SLAC1 in bak1, which was similar to that in $r l k 7$ (Supplementary Figure S2). These results suggest that BAK1 may be required for PIP1-RLK7 signaling in inducing stomatal closure process. In future research, it will be necessary to determine whether BAK1 serves as a co-receptor of RLK7 in perceiving the PIP1 ligand. In plants, MPKs widely function in various processes of plant development or in response to biotic and abiotic stresses (Rodriguez et al., 2010; Lee et al., 2016). Arabidopsis genome encodes 20 MPKs and among these, MPK3 and MPK6 function in flg22-induced stomatal closure (Montillet et al., 2013). In addition, MPK3 and MPK6 are involved in stomatal immunity and MPK3/MPK6 cascade-induced stomatal closure is related to malate/citrate metabolism (Su et al., 2017). Recently, it has been shown that MPK3 and MPK6 also serve as the downstream components in peptide CLE9-induced stomatal closure (Zhang et al., 2019). PIP1 also can induce the phosphorylation of MPK3 and MPK6 in an RLK7-dependent manner (Hou et al., 2014). In our study, we demonstrated that both mutant $m p k 3$ and $m p k 6$ were insensitive to PIP1 (Figure 3), which indicates that PIP1-RLK7 possibly stimulates stomatal closure through the activation of $M P K 3 / M P K 6$-mediated pathways. But it is required to further investigate how PIP1-RLK7 influences MPK3/MPK6 and then induces stomatal closure.

There are multiple signaling elements have been discovered which play critical roles for ABA-induced stomatal closure. We hypothesize that some key signaling elements may also play roles in PIP1-induced activation of S-type anion channels. Therefore, we examined the possible functions of several signaling molecules including a SnRK2-type kinase, OST1. OST1 has been demonstrated to contribute in flg22-FLS2 signaling, and it is required to activate the guard cell S-type anion channels to induce stomatal closure (Melotto et al., 2006; Koers et al., 2011; Ye et al., 2015). Therefore, we expected that PIP1-RLK7 signaling may also require OST1 to initiate stomatal closure. The stomatal experiment result indicated that, to some extent, OST1 mediated the regulation of PIP1-induced stomatal closure (Figure 3). Our finding suggests that PIP1-mediated stomatal response and ABAinduced stomatal closure overlap in common downstream components including OST1. BAK1 can serve as an upstream activator of OST1 and can form a complex with OST1 in response to ABA in guard cells (Shang et al., 2016). In addition, BAK1 has been shown to play a role in PIP1-RLK7 signaling. Accordingly, we assume that BAK1 may also function by interacting with OST1 in PIP1-RLK7 pathway which is similar to in ABA-signaling. In future study, we would like to confirm the relationship between $B A K 1$ and OST1 in PIP1 signaling. Furthermore, ROS have been considered as crucial secondary messages in ABA-induced stomatal closure and OST1 


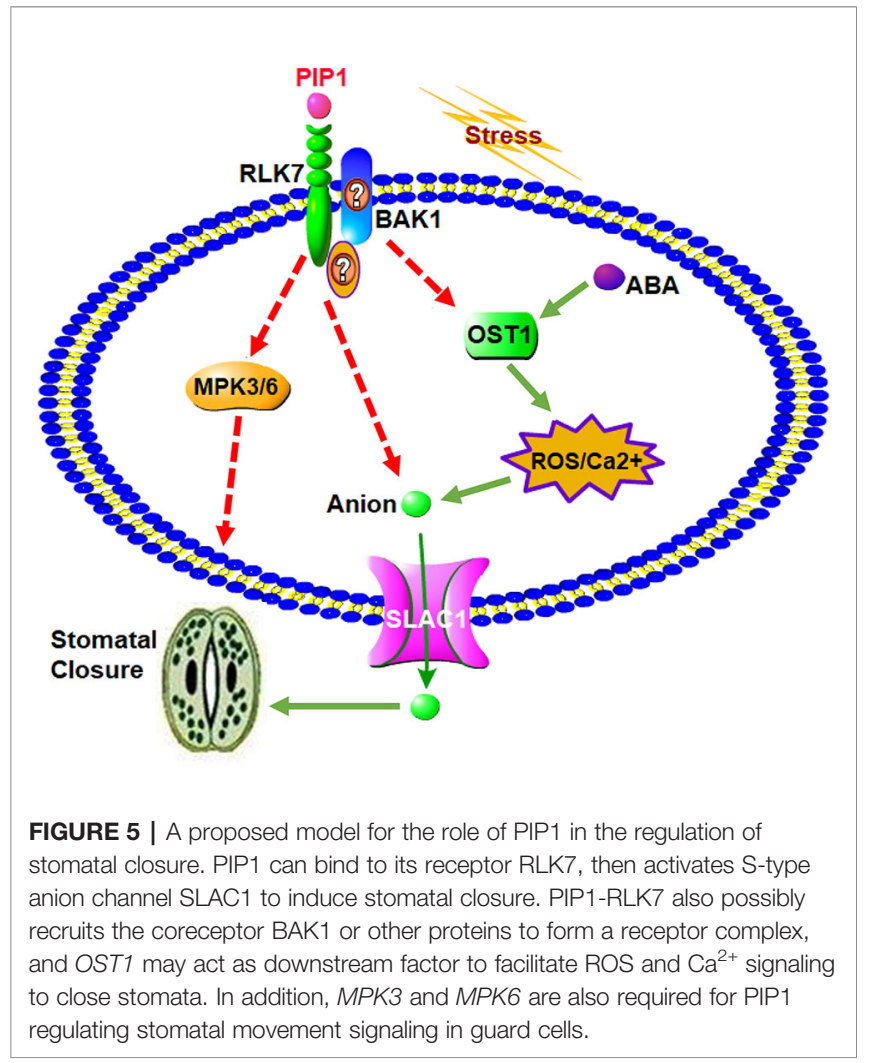

catalyzes ROS production $\left(\mathrm{H}_{2} \mathrm{O}_{2}\right)$ mediated by NADPH oxidase (RbohD and RbohF) (Brandt et al., 2012). In our study, we detected double mutant $r b o h D / F$ was insensitive to PIP1 for closing stomata (Figure 4). The data suggest that ROS generation is required for PIP1-induced stomatal closure. Besides, the study (Kwak et al., 2003) has been reported that $\mathrm{H}_{2} \mathrm{O}_{2}$ application in guard cells activates $\mathrm{Ca}^{2+}$ channels and produces concurrent cytosolic $\mathrm{Ca}^{2+}$ increase. Here, the results indicated that PIP1 could also activate $\mathrm{Ca}^{2+}$-permeable channels in RLK7-dependent manner (Figures 4B-D), suggesting that $\mathrm{Ca}^{2+}$ signal may participate in PIP1-induced stomatal closure.

In conclusion, a possible working model for the role of secreted peptide PIP1 in the regulation of stomatal closure is presented in Figure 5. The function of PIP1-induced stomatal closure depends on the perception by its receptor RLK7, then further activates S-type anion channel SLAC1 to lead to stomatal closure. On one hand, RLK7 may share a downstream component, OST1, with the wellstudied guard cell ABA pathway by recruiting BAK1 or other unknown factors. OST1 can induce the production of ROS, and ROS further activate $\mathrm{Ca}^{2+}$ channels. These factors ultimately result in the activation of anion channel SLAC1 to promote stomatal closure. On the other hand, we found that PIP1-mediated regulation of stomatal aperture requires $M P K 3 / M P K 6$, which serve as downstream mediators. Collectively, our study further reveals that PIP1 peptides can regulate stomatal aperture and explains the possible functional mechanism. It not only connects peptide signaling with stomatal regulation, but also illustrates that PIP1 may function in stress response by closing stomata. Nevertheless, there are many specific questions remaining to be answered and further work will be carried out to clarify molecular mechanism of
PIP1-induced stomatal closure. We also need to further investigate how PIP1 influences $M P K 3 / M P K 6$ pathway and the relationship between RLK7 and BAK1 will be confirmed by protein-protein interaction approaches (such as Co-IP). In addition, we cannot confirm if OST1 mediates PIP1-signaling in BAK1-dependent manner, or there are other factors participating in signal transduction from PIP1 perception to the activation of SLAC1. We would like to study in future by employing multipronged approaches.

\section{DATA AVAILABILITY STATEMENT}

All datasets generated for this study are included in the article/ Supplementary Material.

\section{AUTHOR CONTRIBUTIONS}

WZ and DC are corresponding authors. JS and WD designed and performed the major experiments. JS analyzed the results and wrote the manuscript. LZ performed few experiments and interpreted the data. MW, BA, DC, and XZ designed a few experiments and analyzed some data. WZ conceived and designed the study, interpreted the results, and corrected the manuscript. All authors contributed to the article and approved the submitted version.

\section{FUNDING}

This work was financially supported by Distinguished Young Scholar of Shandong University (61200088963137), Natural Science Foundation of Shandong Province (ZR201807100168) and Cooperation project on nutritional value and health care function of special vegetable for pregnant women and infants (61200012001902).

\section{ACKNOWLEDGMENTS}

We thank Shuguo Hou (Shandong Jianzhu University) for providing the seeds of PIP1 over-expression lines, $r l k 7, b a k 1, b i k 1$, $m p k 3$, and $m p k 6$. We are also thankful to Yan Zhang (Shandong Agricultural University) and Yongfei Wang (Shanghai Institute of Plant Physiology and Ecology, Chinese Academy of Sciences) for the gift of the double mutant $\mathrm{rbohD} / \mathrm{F}$ and SLAC1 mutants (slac1-1 and slac1-3) respectively.

\section{SUPPLEMENTARY MATERIAL}

The Supplementary Material for this article can be found online at: https://www.frontiersin.org/articles/10.3389/fpls.2020.01029/ full\#supplementary-material

FIGURE S1 | (A) The Pst DC3118 induction on SLAC1 transcription in Col-0 seedlings. Asterisks indicate significant differences between means ( ${ }^{*}: \mathrm{P}<0.05$ ). (B) Stomatal closure experiments in slac1-1 and slac1-3 mutants in response to Pst DC3118 (the final concentration of $10^{8} \mathrm{cfu} / \mathrm{ml}$ ). Error bars indicate SE for three 
independent biological replicates. Different letters represent significant differences between groups after one-way ANOVA $(P$ value $<0.05)$.

FIGURE S2 | The SLAC1 transcription in Col-0 and rlk7, bak1, bik1 mutants upon PIP1 treatment. Asterisks indicate significant differences between means ( $P<0.05$ ).

\section{REFERENCES}

Acharya, B. R., Jeon, B. W., Zhang, W., and Assmann, S. M. (2013). Open stomata 1 (OST1) is limiting in abscisic acid responses of Arabidopsis guard cells. New Phytol. 200, 1049-1063. doi: 10.1111/nph.12469

Bergmann, D. C., and Sack, F. D. (2007). Stomatal development. Annu. Rev. Plant Biol. 58, 163-181. doi: 10.1146/annurev.arplant.58.032806.104023

Blatt, M. R., Brodribb, T. J., and Torii, K. U. (2017). Small pores with a big impact. Plant Physiol. 174, 467-469. doi: 10.1104/pp.17.00642

Brandt, B., Brodsky, D. E., Xue, S., Negi, J., Iba, K., Kangasjärvi, J., et al. (2012). Reconstitution of abscisic acid activation of SLAC1 anion channel by CPK6 and OST1 kinases and branched ABI1 PP2C phosphatase action. Proc. Natl. Acad. Sci. U.S.A. 109, 10593-10598. doi: 10.1073/pnas.1116590109

Chen, D. H., Liu, H. P., and Li, C. L. (2019). Calcium-dependent protein kinase CPK9 negatively functions in stomatal abscisic acid signaling by regulating ion channel activity in Arabidopsis. Plant Mol. Biol. 99, 113-122. doi: 10.1007/ s11103-018-0805-y

Chinchilla, D., Zipfel, C., Robatzek, S., Kemmerling, B., Nürnberger, T., Jones, J. D., et al. (2007). A flagellin-induced complex of the receptor FLS2 and BAK1 initiates plant defence. Nature 448, 497-500. doi: 10.1038/nature05999

Cutler, S. R., Rodriguez, P. L., Finkelstein, R. R., and Abrams, S. R. (2010). Abscisic acid: emergence of a core signaling network. Annu. Rev. Plant Biol. 61, 651679. doi: 10.1146/annurev-arplant-042809-112122

Czyzewicz, N., Yue, K., Beeckman, T., and De Smet, I. (2013). Message in a bottle: small signalling peptide outputs during growth and development. J. Exp. Bot. 64, 5281-5296. doi: 10.1093/jxb/ert283

Dong, W., Wang, Y., and Takahashi, H. (2019). CLE-CLAVATA1 signaling pathway modulates lateral root development under sulfur deficiency. Plants 8, 103. doi: $10.3390 /$ plants 8040103

Geiger, D., Scherzer, S., Mumm, P., Stange, A., Marten, I., Bauer, H., et al. (2009). Activity of guard cell anion channel SLAC1 is controlled by drought-stress signaling kinase-phosphatase pair. Proc. Natl. Acad. Sci. U.S.A. 106, 2142521430. doi: 10.1073/pnas.0912021106

Guzel Deger, A., Scherzer, S., Nuhkat, M., Kedzierska, J., Kollist, H., Brosché, M., et al. (2015). Guard cell SLAC1-type anion channels mediate flagellin-induced stomatal closure. New Phytol. 208, 162-173. doi: 10.1111/nph.13435

Hou, S. G., Wang, X., Chen, D. H., Yang, X., Wang, M., Turrà, D., et al. (2014). The secreted peptide PIP1 amplifies immunity through receptor-like kinase 7. PloS Pathog. 10, e1004331. doi: 10.1371/journal.ppat.1004710

Hou, S. G., Shen, H. X., and Shao, H. W. (2019). PAMP-induced peptide 1 cooperates with salicylic acid to regulate stomatal immunity in Arabidopsis thaliana. Plant Signal Behav. 14, 1666657. doi: 10.1080/15592324.2019.1666657

Joshi-Saha, A., Valon, C., and Leung, J. (2011). A brand new START: abscisic acid perception and transduction in the guard cell. Science 4, re4. doi: 10.1126/ scisignal.2002164

Kim, T. H., Böhmer, M., Hu, H., Nishimura, N., and Schroeder, J. I. (2010). Guard cell signal transduction network: advances in understanding abscisic acid, $\mathrm{CO}_{2}$, and $\mathrm{Ca}^{2+}$ signaling. Annu. Rev. Plant Biol. 61, 561-591. doi: 10.1146/annurevarplant-042809-112226

Koers, S., Guzel-Deger, A., Marten, I., and Roelfsema, M. R. (2011). Barley mildew and its elicitor chitosan promote closed stomata by stimulating guard-cell Stype anion channels. Plant J. 68, 670-680. doi: 10.1111/j.1365-313X.2011.04719.x

Kollist, H., Nuhkat, M., and Roelfsema, M. R. (2014). Closing gaps: linking elements that control stomatal movement. New Phytol. 203, 44-62. doi: $10.1111 /$ nph.12832

Kwak, J. M., Mori, I. C., Pei, Z. M., Leonhardt, N., Torres, M. A., Dangl, J. L., et al. (2003). NADPH oxidase AtrbohD and AtrbohF genes function in ROSdependent ABA signaling in Arabidopsis. EMBO J. 22, 2623-2633. doi: 10.1093/ $\mathrm{emboj} / \mathrm{cdg} 277$
FIGURE S3 | (A) The qPCR-based assessment of PIP1 transcription in Col-0 and the over-expression lines PIP1-OE and PIP1-OE2. (B) Stomatal aperture in PIP1OE2 by treating with Pst DC3118 (the final concentration of $10^{8} \mathrm{cfu} / \mathrm{ml}$ ). Different letters represent significant differences between groups after one-way ANOVA ( $P$ value $<0.05$ )

Lee, S. C., and Luan, S. (2012). ABA signal transduction at the crossroad of biotic and abiotic stress responses. Plant Cell Environ. 35, 53-60. doi: 10.1111/j.13653040.2011.02426.x

Lee, S. C., Lim, C. W., Lan, W., He, K., and Luan, S. (2013). ABA signaling in guard cells entails a dynamic protein-protein interaction relay from the PYL-RCAR family receptors to ion channels. Mol. Plant 6, 528-538. doi: 10.1093/mp/ sss078

Lee, Y., Kim, Y. J., Kim, M. H., and Kwak, J. M. (2016). MAPK cascades in guard cell signal transduction. Front. Plant Sci. 7, 80. doi: 10.3389/fpls.2016.00080

Li, J., Wang, X. Q., Watson, M. B., and Assmann, S. M. (2000). Regulation of abscisic acid-induced stomatal closure and anion channels by guard cell AAPK kinase. Science 287, 300-303. doi: 10.1126/science.287.5451.300

Li, L., Li, M., Yu, L., Zhou, Z., Liang, X., Liu, Z., et al. (2014). The FLS2-associated kinase BIK1 directly phosphorylates the NADPH oxidase RbohD to control plant immunity. Cell Host. Microbe 15, 329-338. doi: 10.1016/j.chom.2014.02.009

Li, C. L., Wang, M., Wu, X. M., Chen, D. H., Lv, H. J., Shen, J. L., et al. (2016). THI1, a thiamine thiazole synthase, interacts with $\mathrm{Ca}^{2+}$-dependent protein kinase CPK33 and modulates the S-type anion channels and stomatal closure in Arabidopsis. Plant Physiol. 170, 1090-1104. doi: 10.1104/pp.15.01649

Liu, Z., Wu, Y., Yang, F., Zhang, Y., Chen, S., Xie, Q., et al. (2013). BIK1 interacts with PEPRs to mediate ethylene-induced immunity. Proc. Natl. Acad. Sci. U.S.A. 110, 6205-6210. doi: 10.1073/pnas.1215543110

Lu, D., Wu, S., Gao, X., Zhang, Y., Shan, L., and He, P. (2010). A receptor-like cytoplasmic kinase, BIK1, associates with a flagellin receptor complex to initiate plant innate immunity. Proc. Natl. Acad. Sci. U.S.A. 107, 496-501. doi: 10.1073/pnas.0909705107

Mao, G., Meng, X., Liu, Y., Zheng, Z., Chen, Z., and Zhang, S. (2011). Phosphorylation of a WRKY transcription factor by two pathogenresponsive MAPKs drives phytoalexin biosynthesis in Arabidopsis. Plant Cell. 23, 1639-1653. doi: 10.1105/tpc.111.084996

Melotto, M., Underwood, W., Koczan, J., Nomura, K., and He, S. Y. (2006). Plant stomata function in innate immunity against bacterial invasion. Cell 126, 969980. doi: 10.1016/j.cell.2006.06.054

Meng, X., Xu, J., He, Y., Yang, K. Y., Mordorski, B., Liu, Y., et al. (2013). Phosphorylation of an ERF transcription factor by Arabidopsis MPK3/ MPK6 regulates plant defense gene induction and fungal resistance. Plant Cell. 25, 1126-1142. doi: 10.1105/tpc.112.109074

Merilo, E., Jalakas, P., Laanemets, K., Mohammadi, O., Hõrak, H., Kollist, H., et al. (2015). Abscisic acid transport and homeostasis in the context of stomatal regulation. Mol. Plant 8, 1321-1333. doi: 10.1016/j.molp.2015.06.006

Monaghan, J., and Zipfel, C. (2012). Plant pattern recognition receptor complexes at the plasma membrane. Curr. Opin. Plant Biol. 15, 349-357. doi: 10.1016/ j.pbi.2012.05.006

Montillet, J. L., Leonhardt, N., Mondy, S., Tranchimand, S., Rumeau, D., Boudsocq, M., et al. (2013). An abscisic acid-independent oxylipin pathway controls stomatal closure and immune defense in Arabidopsis. PloS Biol. 11, e1001513. doi: 10.1371/ journal.pbio.1001513

Moscatiello, R., Mariani, P., Sanders, D., and Maathuis, F. J. (2006). Transcriptional analysis of calcium-dependent and calcium-independent signalling pathways induced by oligogalacturonides. J. Exp. Bot. 57, 28472865. doi: 10.1093/jxb/erl043

Munemasa, S., Hirao, Y., Tanami, K., Mimata, Y., Nakamura, Y., and Murata, Y. (2019). Ethylene inhibits methyl jasmonate-induced stomatal closure by modulating guard cell slow-type anion channel activity via the OPEN STOMATA 1/SnRK2.6 kinase-independent pathway in Arabidopsis. Plant Cell Physiol. 60, 2263-2271. doi: 10.1093/pcp/pcz121

Murata, Y., Mori, I. C., and Munemasa, S. (2015). Diverse stomatal signaling and the signal integratio mechanism. Annu. Rev. Plant Biol. 66, 369-392. doi: 10.1146/ annurev-arplant-043014-114707 
Murphy, E., Smith, S., and De Smet, I. (2012). Small signaling peptides in Arabidopsis development: how cells communicate over a short distance. Plant Cell. 24, 3198-3217. doi: 10.1105/tpc.112.099010

Okamoto, M., Peterson, F. C., Defries, A., Park, S. Y., Endo, A., Nambara, E., et al. (2013). Activation of dimeric ABA receptors elicits guard cell closure, ABAregulated gene expression, and drought tolerance. Proc. Natl. Acad. Sci. U.S.A. 110, 12132-12137. doi: 10.1073/pnas.1305919110

Pei, Z. M., Kuchitsu, K., Ward, J. M., Schwarz, M., and Schroeder, J. I. (1997). Differential abscisic acid regulation of guard cell slow anion channels in Arabidopsis wild-type and abil and abi2 mutants. Plant Cell. 9, 409-423. doi: $10.1105 /$ tpc.9.3.409

Pei, Z. M., Murata, Y., Benning, G., Thomine, S., Klüsener, B., Allen, G. J., et al. (2000). Calcium channels activated by hydrogen peroxide mediate abscisic acid signalling in guard cells. Nature 406, 731-734. doi: 10.1038/35021067

Pitorre, D., Llauro, C., Jobet, E., Guilleminot, J., Brizard, J. P., Delseny, M., et al. (2010). RLK7, a leucine-rich repeat receptor-like kinase, is required for proper germination speed and tolerance to oxidative stress in Arabidopsis Thaliana. Planta 232, 1339-1353. doi: 10.1007/s00425-010-1260-4

Purohit, S., Kumar, G. P., Laloraya, M., and Laloraya, M. M. (1994). Involvement of superoxide radical in signal transduction regulating stomatal movements. Biochem. Biophys. Res. Commun. 205, 30-37. doi: 10.1006/bbrc.1994.2625

Qu, X., Cao, B., Kang, J., Wang, X., Han, X., Jiang, W., et al. (2019). Fine-tuning stomatal movement through small signaling peptides. Front. Plant Sci. 10, 69. doi: $10.3389 /$ fpls.2019.00069

Raghavendra, A. S., Gonugunta, V. K., Christmann, A., and Grill, E. (2010). ABA perception and signalling. Trends Plant Sci. 15, 395-401. doi: 10.1016/ j.tplants.2010.04.006

Rodriguez, M. C., Petersen, M., and Mundy, J. (2010). Mitogen-activated protein kinase signaling in plants. Annu. Rev. Plant Biol. 61, 621-649. doi: 10.1146/ annurev-arplant-042809-112252

Shang, Y., Dai, C., Lee, M. M., Kwak, J. M., and Nam, K. H. (2016). BRI1associated receptor kinase 1 regulates guard cell ABA signaling mediated by Open Stomata 1 in Arabidopsis. Mol. Plant 9, 447-460. doi: 10.1016/j.molp. 2015.12.014

Shen, J. L., Li, C. L., Wang, M., He, L. L., Lin, M. Y., Chen, D. H., et al. (2017). Mitochondrial pyruvate carrier 1 mediates abscisic acid-regulated stomatal closure and the drought response by affecting cellular pyruvate content in Arabidopsis thaliana. BMC Plant Biol. 17, 217. doi: 10.1186/s12870-017-1175-3

Singh, R., Parihar, P., Singh, S., Mishra, R. K., Singh, V. P., and Prasad, S. M. (2017). Reactive oxygen species signaling and stomatal movement: current updates and future perspectives. Redox Biol. 11, 213-218. doi: 10.1016/j.redox .2016 .11 .006

Speth, E. B., Melotto, M., Zhang, W., Assmann, S. M., and He, S. Y. (2009). Crosstalk in pathogen and hormonal regulation of guard cell signaling. Signal Crosstalk in Plant Stress Responses. doi: 10.1002/9780813805931.ch5

Su, J. B., Zhang, M. M., Zhang, L., Sun, T. F., Liu, Y. D., Lukowitz, W., et al. (2017). Regulation of stomatal immunity by interdependent functions of a pathogenresponsive MPK3/MPK6 cascade and abscisic acid. Plant Cell. 29, 526-542. doi: $10.1105 /$ tpc. 16.00577

Sun, Y., Li, L., Macho, A. P., Han, Z., Hu, Z., Zipfel, C., et al. (2013). Structural basis for flg22-induced activation of the Arabidopsis FLS2-BAK1 immune complex. Science 342, 624-628. doi: 10.1126/science.1243825

Sun, L. R., Yue, C. M., and Hao, F. S. (2019). Update on roles of nitric oxide in regulating stomatal closure. Plant Signal Behav. 1, 1-4. doi: 10.1080/15592 324.2019.1649569

Suzuki, N., Miller, G., Morales, J., Shulaev, V., Torres, M. A., and Mittler, R. (2011). Respiratory burst oxidases: the engines of ROS signaling. Curr. Opin. Plant Biol. 14, 691-699. doi: 10.1016/j.pbi.2011.07.014

Takahashi, F., Suzuki, T., Osakabe, Y., Betsuyaku, S., Kondo, Y., Dohmae, N., et al. (2018). A small peptide modulates stomatal control via abscisic acid in longdistance signalling. Nature 556, 235-238. doi: 10.1038/s41586-018-0009-2

Vahisalu, T., Kollist, H., Wang, Y. F., Nishimura, N., Chan, W. Y., Valerio, G., et al. (2008). SLAC1 is required for plant guard cell S-type anion channel function in stomatal signalling. Nature 452, 487-491. doi: 10.1038/nature06608
Vahisalu, T., Puzõrjova, I., Brosché, M., Valk, E., Lepiku, M., Moldau, H., et al. (2010). Ozone-triggered rapid stomatal response involves the production of reactive oxygen species, and is controlled by SLAC1 and OST1. Plant J. 62, 442-453. doi: 10.1111/j.1365-313X.2010.04159.x

Wang, G., and Fiers, M. (2010). CLE peptide signaling during plant development. Protoplasma 240, 33-43. doi: 10.1007/s00709-009-0095-y

Wang, X. Q., Ullah, H., Jones, A. M., and Assmann, S. M. (2001). G protein regulation of ion channels and abscisic acid signaling in Arabidopsis guard cells. Science 292, 2070-2072. doi: 10.1126/science.1059046

Wang, X., Kota, U., He, K., Blackburn, K., Li, J., Goshe, M. B., et al. (2008). Sequential transphosphorylation of the BRI1/BAK1 receptor kinase complex impacts early events in brassinosteroid signaling. Dev. Cell. 15, 220-235. doi: 10.1016/j.devcel.2008.06.011

Wang, Y., Chen, Z. H., Zhang, B., Hills, A., and Blatt, M. R. (2013). PYR/PYL/ RCAR abscisic acid receptors regulate $\mathrm{K}^{+}$and $\mathrm{Cl}^{-}$channels through reactive oxygen species-mediated activation of $\mathrm{Ca}^{2+}$ channels at the plasma membrane of intact Arabidopsis guard cells. Plant Physiol. 163, 566-577. doi: 10.1104/ pp.113.219758

Wang, G., Zhang, G., and Wu, M. (2016). CLE peptide signaling and crosstalk with phytohormones and environmental stimuli. Front. Plant Sci. 6, 1211. doi: 10.3389/ fpls.2015.01211

Xu, J., Xie, J., Yan, C. F., Zou, X. Q., Ren, D. T., and Zhang, S. Q. (2014). A chemical genetic approach demonstrates that MPK3/MPK6 activation and NADPH oxidase-mediated oxidative burst are two independent signaling events in plant immunity. Plant J. 77, 222-234. doi: 10.1111/tpj.12382

Yang, D. L., Shi, Z., Bao, Y., Yan, J., Yang, Z., Yu, H., et al. (2017). Calcium pumps and interacting BON1 protein modulate calcium signature, stomatal closure, and plant immunity. Plant Physiol. 175, 424-437. doi: 10.1104/pp.17.00495

Ye, W., Adachi, Y., Munemasa, S., Nakamura, Y., Mori, I. C., and Murata, Y. (2015). Open Stomata 1 kinase is essential for yeast elicitor-induced stomatal closure in Arabidopsis. Plant Cell Physiol. 56, 1239-1248. doi: 10.1093/pcp/pcv051

Yu, Y., Chakravorty, D., and Assmann, S. M. (2018). The G protein $\beta$-subunit, AGB1, interacts with FERONIA in RALF1-regulated stomatal movement. Plant Physiol. 176, 2426-2440. doi: 10.1104/pp.17.01277

Zhang, W., Nilson, S. E., and Assmann, S. M. (2008). Isolation and whole-cell patch clamping of Arabidopsis guard cell protoplasts. CSH Protoc. 2008, pdb.prot5014. doi: 10.1101/pdb.prot5014

Zhang, J., Li, W., Xiang, T. T., Liu, Z. X., Laluk, K., Ding, X. J., et al. (2010). Receptor-like cytoplasmic kinases integrate signaling from multiple plant immune receptors and are targeted by a Pseudomonas syringae effector. Cell Host. Microbe 7, 290-301. doi: 10.1016/j.chom.2010.03.007

Zhang, J., Wang, N., Miao, Y., Hauser, F., McCammon, J. A., Rappel, W. J., et al. (2018). Identification of SLAC1 anion channel residues required for $\mathrm{CO}_{2} /$ bicarbonate sensing and regulation of stomatal movements. Proc. Natl. Acad. Sci. U.S.A. 115, 11129-11137. doi: 10.1073/pnas.1807624115

Zhang, L., Shi, X., Zhang, Y., Wang, J., Yang, J., Ishida, T., et al. (2019). CLE9 peptide-induced stomatal closure is mediated by abscisic acid, hydrogen peroxide, and nitric oxide in Arabidopsis Thaliana. Plant Cell Environ. 42, 1033-1044. doi: 10.1111/pce.13475

Zheng, X., Kang, S., Jing, Y., Ren, Z., Li, L., Zhou, J. M., et al. (2018). Dangerassociated peptides close stomata by OST1-independent activation of anion channels in guard cells. Plant Cell. 30, 1132-1146. doi: 10.1105/tpc.17.00701

Conflict of Interest: The authors declare that the research was conducted in the absence of any commercial or financial relationships that could be construed as a potential conflict of interest.

Copyright $\odot 2020$ Shen, Diao, Zhang, Acharya, Wang, Zhao, Chen and Zhang. This is an open-access article distributed under the terms of the Creative Commons Attribution License (CC BY). The use, distribution or reproduction in other forums is permitted, provided the original author(s) and the copyright owner(s) are credited and that the original publication in this journal is cited, in accordance with accepted academic practice. No use, distribution or reproduction is permitted which does not comply with these terms. 